\title{
Anxiety, Depression and Post Traumatic Stress Disorder after critical illness: a UK- wide prospective cohort study
}

\author{
Robert Hatch ${ }^{1,7}$, Duncan Young ${ }^{2,7}$, Vicki Barber ${ }^{3}$, John Griffiths ${ }^{4}$, David A. Harrison ${ }^{5}$ and Peter Watkinson ${ }^{6,7^{*}}$ (D)
}

\begin{abstract}
Background: Survivors of intensive care are known to be at increased risk of developing longer-term psychopathology issues. We present a large UK multicentre study assessing the anxiety, depression and posttraumatic stress disorder (PTSD) caseness in the first year following discharge from an intensive care unit (ICU).

Methods: Design: prospective multicentre follow-up study of survivors of ICU in the UK. Setting: patients from 26 ICUs in the UK.

Inclusion criteria: patients who had received at least $24 \mathrm{~h}$ of level 3 ICU care and were 16 years of age or older. Interventions: postal follow up: Hospital Anxiety and Depression Score (HADS) and the Post-Traumatic Stress Disorder (PTSD) Check List-Civilian (PCL-C) at 3 and 12 months following discharge from ICU. Main outcome measure: caseness of anxiety, depression and PTSD, 2-year survival.
\end{abstract}

Results: In total, 21,633 patients admitted to ICU were included in the study. Postal questionnaires were sent to 13,155 survivors; of these $38 \%$ (4943/13155) responded and 55\% (2731/4943) of respondents passed thresholds for one or more condition at 3 or 12 months following discharge. Caseness prevalence was $46 \%, 40 \%$ and $22 \%$ for anxiety, depression and PTSD respectively; 18\% (870/4943 patients) met the caseness threshold for all three psychological conditions. Patients with symptoms of depression were $47 \%$ more likely to die during the first 2 years after discharge from ICU than those without (HR 1.47, CI 1.19-1.80).

Conclusions: Over half of those who respond to postal questionnaire following treatment on ICU in the UK reported significant symptoms of anxiety, depression or PTSD. When symptoms of one psychological disorder are present, there is a $65 \%$ chance they will co-occur with symptoms of one of the other two disorders. Depression following critical illness is associated with an increased mortality risk in the first 2 years following discharge from ICU.

Trial registration: ISRCTN Registry, ISRCTN69112866. Registered on 2 May 2006.

Keywords: Anxiety, Depression, Post-traumatic stress disorder, PTSD, Post-traumatic stress disorder civilian checklist, PCL-C, Post-Intensive Care Syndrome, PICS, Questionnaire, Postal, Intensive care, Critical care, Survivors, Multicentre study, Critical illness, Outcome assessment

\footnotetext{
* Correspondence: peter.watkinson@ndcn.ox.ac.uk

${ }^{6}$ Associate Professor of Intensive Care Medicine, Oxford University Hospitals

NHS Trust, Oxford, UK

${ }^{7}$ Kadoorie Centre for Critical Care Research and Education, University of

Oxford, Level 3, John Radcliffe Hospital, Headley Way, Oxford OX3 9DU, UK

Full list of author information is available at the end of the article
}

(c) The Author(s). 2018 Open Access This article is distributed under the terms of the Creative Commons Attribution 4.0 International License (http://creativecommons.org/licenses/by/4.0/), which permits unrestricted use, distribution, and reproduction in any medium, provided you give appropriate credit to the original author(s) and the source, provide a link to the Creative Commons license, and indicate if changes were made. The Creative Commons Public Domain Dedication waiver (http://creativecommons.org/publicdomain/zero/1.0/) applies to the data made available in this article, unless otherwise stated. 


\section{Background}

Survivors of critical illness are at risk of experiencing significant physical, cognitive and psychological issues after discharge [1]. New or worsening impairment in physical, cognitive or mental health status following treatment on an intensive care unit (ICU) is known as post-intensive care syndrome (PICS) [2]. The psychopathological components of PICS are estimated to occur in up to a third of survivors of ICU treatment [1]. The major psychological conditions described are anxiety, depression and post-traumatic stress disorder (PTSD).

Symptoms of anxiety following critical illness occur in $25-46 \%$ of patients in the $3-14$ months following discharge from an ICU [3]. Post ICU anxiety is associated with psychiatric symptoms, memories and delusions [46]. Unlike the general population, where anxiety is more common in women aged 30-44 years [7], no association with age, sex, disease severity or length of stay has been found in patients treated on an ICU. This difference may reflect a causal association or the underpowered nature of the individual studies (where the largest previous study included 255 patients [8]).

Depressive symptoms occur in around $29 \%$ of survivors at 3, 6 and 12 months post ICU discharge [3]. Depressive illness following intensive care is associated with symptoms of psychological distress (anxiety, stress and anger). The Hospital Anxiety and Depression Scale (HADS) is the most commonly used (and validated) tool; however, the most recent meta-analysis identified only 387 unique patients with HADS data at 3 and 6 months post discharge and a further 412 at 6 and 12 months [9]. Like anxiety, there was no association with age or sex, in contrast to findings in general populations [9]. In addition, there was no correlation with ICU length of stay or illness severity.

A recent meta-analysis (2015) estimated PTSD symptom prevalence at $17-34 \%$ at 12 months post-ICU discharge [3]. Risk factors for the development of post-ICU PTSD include the presence of pre-ICU anxiety and depression.

The risk of mortality in survivors of critical illness is 3.4 times greater than that of the general population over the 5 years following discharge [10]. Mortality remains greater than that of the general population for up to 4 years following ICU admission. There is a known association between depression and an increased risk of death, both in the general population $[11,12]$ and specific subgroups of patients with illness-comorbid depression. Conversely, anxiety and/or PTSD have been associated with a decreased mortality hazard [11]. Whether the presence of psychopathology affects mortality following treatment on an ICU overall is unknown.

Previous studies of psychopathology after critical illness are limited by their size. In many cases they are performed in specific sub-populations, conditions or as part of a randomised controlled trial (RCT) $[3,9,13]$. Survivors of critical illness are heterogeneous in terms of illness severity, treatment and long-term prognosis. Few studies have concurrently assessed symptoms of anxiety, depression and PTSD in a general adult ICU population. ICU-specific conditions such as acute respiratory depression syndrome (ARDS) are associated with poorer longer-term outcome and a high incidence of psychopathology [13]. However, these diagnoses represent the minority of overall ICU admissions.

\section{Objective}

We postulated that illness-comorbid depression is independently associated with survival, when other known associations such as age and illness severity are considered. We undertook a large multicentre postal survey of all patients admitted to participating UK general adult ICUs. Our objective was to describe the pattern of psychopathology occurring in survivors at 3 and 12 months following discharge from ICU and to assess any association with mortality.

\section{Methods}

This study is reported following the Strengthening of Reporting in Observational studies in Epidemiology (STROBE) guidance [14].

\section{Study design}

The Intensive Care Outcomes Network study (ICON) was a UK multicentre prospective cohort study assessing heath related quality of life (HRQoL) and caseness of anxiety, depression and PTSD, by postal questionnaire, following at least $24 \mathrm{~h}$ treatment on an ICU. Caseness is the degree to which the accepted standardised diagnostic criteria for a given condition are applicable to a given patient. A questionnaire study assesses the self-reported symptom burden consistent with a specific disorder but cannot be considered diagnostic. The trial was registered ISRCTN69112866 (assigned 2 May 2006) and the study protocol has been published [15]. This study was conducted in three consecutive phases (summarised in Additional file 1). Ethical approval for phases 1 and 2 was granted by Oxfordshire Research Ethics Committee B (REC 06/ Q1605/17). Ethical approval for phase 3 was granted by National Research Ethics Service - South Central Berkshire (REC 11/SC/0172). The ICON study had Section 60 of the Health and Social Care Act 2001 (subsequently Section 251 of the National Health Service (NHS) Act 2006) approval (PIAG 2-05(e)/2006). This granted permission to record details of all admissions meeting the inclusion criteria at participating ICUs. 


\section{Setting}

Phases 1 and 2 took place in the same 26 UK ICUs (1 university hospital, 6 university-affiliated hospitals and 19 district general hospitals) and postal questionnaires were sent at 3, 12 and 24 months following discharge form ICU. Phase 1 recruited from November 2006 to May 2008 and recruited 9582 patients. Phase 2 recruited from May 2008 to October 2010 and recruited 18,490 patients as part of an RCT to study the effect of different questionnaire burden on response rate, the results of which have been published [16].

Phase 3 took place between May 2012 and May 2013 in 31 UK ICUs (10 university hospital, 3 university-affiliated hospitals and 18 district general hospitals), and of these 18 recruiting centres are common with phases 1 and 2 . Phase 3 recruited 2876 patients, with postal questionnaires administered at 3 and 12 months post ICU discharge. Questionnaire burden was identical to phase 1 . Where possible patients were approached by a research nurse prior to their discharge from hospital. Patients were followed up by telephone if they later failed to respond to a postal questionnaire.

\section{Participants}

We extracted data from all three phases of the ICON study database, with the exception of group A from the second phase, as this group did not receive psychological instruments by design $[15,16]$. Each phase had identical inclusion and exclusion criteria.

Eligible patients received level 3 care on an ICU (as defined by the Intensive Care Society, London [17]), for at least $24 \mathrm{~h}$ and were 16 years or older at ICU admission. We excluded patients not registered with a general practitioner or of no fixed abode (factors anticipated to prevent follow up in the study). We also excluded patients taking part in another questionnaire follow-up study run by the same research office and in phase 3, those patients who withdrew consent prior to discharge from hospital (as we could not legitimately track their mortality even to hospital discharge). In addition, we excluded those patients who could not be matched to the Intensive Care National Audit \& Research Centre (ICNARC) Case Mix Programme database and patients who were not captured by the ICON study during their incident admission to ICU during their hospital stay (as we were timing questionnaire response from their first exposure to ICU). Patients could withdraw their consent at any point during any phase of the study (by contacting the study office or by returning the survey blank). This resulted in their personal identifying data being purged from the study database, anonymising their record at that point. We did not contact patients following a specific request by their GP.

\section{Variables}

Patients received a letter introducing the study at ICU discharge. The letter explained that they might receive mail from the study team and provided contact details for the study office. Eligible patients received postal questionnaires at 3 and 12 months following discharge from ICU. Each mailing included the HADS (14 questions, 7 depression and 7 anxiety, each scored ordinally 0-3), the Post Traumatic Stress Disorder Check List - Civilian version (PCL-C - 17 questions score ordinally 1-5) and other health related quality of life instruments (see Additional file 1). When there was no response to the first mailing, this was followed by a second postal copy 14 days later. In phases 1 and 2 no response after the second mailing was considered a loss to follow up. In phase 3 , an additional attempt was made to call the patient.

A cut-off score $\geq 8$ for either HADS anxiety or depression scales defined caseness of the respective condition [18]. We applied HADS boundaries for mild, moderate and severe symptoms to those exhibiting caseness [19]. A PCL-C score $\geq 45$ defined PTSD caseness [20].

\section{Data sources and measurement}

We linked participant records with the ICNARC Case Mix Programme [21] to obtain admitting diagnoses, severity of illness scores and to capture previous admissions to ICU during their hospital stay. We checked survival and current registered address with the patient's registered general practitioner (GP) and the National Health Service Summary Care Record [22] before posting each questionnaire pack.

With the exception of those who withdrew consent, we linked participants to the Medical Research Information Service (MRIS) run by NHS Digital. This provided linked mortality data and event notification from the Office of National Statistics (ONS).

\section{Bias}

In phase 1 and 2 , clinical staff gave participants a letter explaining that they would receive ICON documentation in the post at the point of discharge from the ICU. In phase 3 a study nurse aimed to visit the patients between ICU and hospital discharge to introduce the ICON study, where a study nurse was available. Further information was provided and a second visit could be arranged to provide written consent if so wished. Patients could remove their consent at any time.

\section{Study size}

Study size was based on the total number of patients meeting inclusion criteria and admitted to the participating units inside the recruitment period.

\section{Quantitative variables}

For this analysis the primary outcome was the proportion of patients meeting predefined thresholds for caseness of anxiety and depression (using the HADS scale) 
and for PTSD (using PCL-C) at 3 and 12 months following discharge from ICU. Secondary outcome measures were survival at 3,12 and 24 months following discharge from ICU, the proportion of individual patients transitioning these thresholds between time points and the correlation between PTSD, anxiety and depression.

\section{Statistical methods}

Statistical analysis was undertaken using $\mathrm{R}$ Core v3.4.1 [23]. We did not correct for multiple testing. Response was defined as return of a questionnaire with valid written consent. Each instrument was scored in accordance with the author's instructions. Individual responses not meeting these instructions were considered invalid and excluded from further analysis. The proportions of patients meeting the criteria for caseness were calculated for each instrument at each time point. Those meeting the HADS thresholds were further subdivided by symptom severity (mild, moderate, severe).

Population demographics, responses to the individual psychological instruments and change analysis were presented in keeping with the pre-specified data collection plan for the ICON Study. Survival analysis was first performed using the Kaplan-Meier (KM) method. Patients were right censored when we could no longer track their mortality (lost to follow up). This occurred when patients explicitly withdrew their consent (excluding them from being enrolled with MRIS/ONS). Study phases and participants with and without caseness were compared using the log-rank test with $p<0.01$.

We performed ad-hoc Cox proportional hazards modelling to study the effects of depression, anxiety and PTSD caseness on survival, adjusting for known confounders. We verified the assumption of proportional hazards prior to conducting the analysis. The study design meant that censoring of individual subjects did not occur as a result of the disease process. We identified age (as a continuous variable), sex (as a binary variable) and severity of illness Acute Physiology and Chronic Health Evaluation (APACHE) II score as a continuous variable [24]) as potential confounders from the literature $[3,13,25]$. The interpretation of a continuous variable is that for each additional unit increase in the continuous variable the hazard ratio increases by the value reported. The interpretation of a binary variable is the hazard ratio associated with male versus female (sex) or caseness versus no caseness for the other variables.

The effect of these variables on survival was confirmed using univariable Cox proportional hazard analysis. Four multivariable models were then constructed. All included the co-founders identified in the literature. Models 1-3 included each psychological disorder in isolation and model 4 included all three.

\section{Results}

\section{Participants}

We screened 21,633 patients for eligibility across the three phases of the study in November 2006 to May 2013: 19,822 patients satisfied the eligibility requirements. Of these, 3289 (17\%) died during their admission to ICU. Following discharge alive from ICU, 2710 (2710/19,822 (14\%)) of those eligible died in the subsequent 75 days. There were 429 patients $(429 / 13,823$ (3\%)) excluded from follow up by request of their general practitioner (GP) (see the patient flow diagram in Additional file 2).

Of those who were alive at the point of being sent a questionnaire at 3 or 12 months, 4943 patients (4943/ $13,155(38 \%)$ ) completed at least one survey. Of those mailed at 3 months $4809(4809 / 12,777(38 \%))$ completed a survey. Of those mailed at 12 months, 3569 (3569/ $4936(72 \%)$ ) completed a survey (non-respondents and those who actively declined to consent at 3 months were not mailed). In total 2943 patients (22,943/13,155 (22\%)) actively declined to consent (by returning a blank form or contacting the research office at 3 or 12 months).

\section{Descriptive data}

Table 1 shows the demographics, illness severity and organ support for those eligible to receive a postal survey and those responding at either 3 or 12 months. The two groups were remarkably balanced in terms of their baseline demographics/measured confounders. Additional file 3 includes identical demographics with columns for those not responding to the study, explicitly removing consent and responding with and without symptoms of psychopathological issues.

\section{Outcome data}

Figure 1 shows the distribution of HADS anxiety, HADS depression and PCL-C in patients that provided valid responses at both 3 and 12 months. Percentages denote those with and without caseness. At 3 months the caseness prevalence for anxiety, depression and PTSD were $45.7 \%, 41.0 \%$ and $22.0 \%$ respectively. An identical analysis including all those responding at either time point is available in Additional file 4. Responses to individual questions are found in Additional files 5 and 6.

Whilst population prevalence is largely unchanged between 3 and 12 months across all three instruments, $10 \%$ of responders met the threshold for significant symptoms consistent with anxiety or depression at 12 months, who had not fulfilled these criteria when they responded at 3 months (Additional file 7). Similarly, $7 \%$ of responders met the symptom threshold for PTSD by 12 months, who had not when responding at 3 months. Therefore, between one third and one half of the patients meeting the threshold for caseness do so at only one of the two time points. 
Table 1 Baseline demographics, illness severity scores, organ support durations and admission diagnoses for those sent a questionnaire and patients responding

\begin{tabular}{|c|c|c|c|c|}
\hline & Sent a questionnaire & & Responders & \\
\hline Patients (n) & 13,155 & & 4943 & \\
\hline Age (median) [25th-75th] & 63 [47-73] & & 64 [52-73] & \\
\hline Sex (\%male) & $57 \%$ & & $57 \%$ & \\
\hline APACHE I| score (median) [25th-75th] & 15 [11-19] & & 15 [11-19] & \\
\hline ICU length of stay (median) [25th-75th] & $3[2-6]$ & & $3[2-6]$ & \\
\hline Hospital length of stay (median) [25th-75th] & 15 [8-29] & & $15[9-28]$ & \\
\hline Advanced cardiovascular support (\% of patients) & $23 \%$ & & $26 \%$ & \\
\hline Basic cardiovascular support (\% of patients) & $90 \%$ & & $91 \%$ & \\
\hline Advanced respiratory support (\% of patients) & $55 \%$ & & $56 \%$ & \\
\hline Basic respiratory support (\% of patients) & $63 \%$ & & $64 \%$ & \\
\hline Renal support (\% of patients) & $9 \%$ & & $10 \%$ & \\
\hline Neurological support (\% of patients) & $10 \%$ & & $9 \%$ & \\
\hline Liver support (\% of patients) & $0 \%$ & & $0 \%$ & \\
\hline Dermatological support (\% of patients) & $4 \%$ & & $5 \%$ & \\
\hline Gastrointestinal support (\% of patients) & $42 \%$ & & $43 \%$ & \\
\hline Respiratory tract infection & $n=1152$ & $9 \%$ & $n=419$ & $8 \%$ \\
\hline Vascular procedure to major vessel & $n=881$ & $7 \%$ & $n=399$ & $8 \%$ \\
\hline Large bowel tumour & $n=695$ & $5 \%$ & $n=315$ & $6 \%$ \\
\hline Self-poisoning & $n=572$ & $4 \%$ & $n=117$ & $2 \%$ \\
\hline Septicaemia and septic shock & $n=431$ & $3 \%$ & $n=173$ & $3 \%$ \\
\hline Acute renal failure & $n=473$ & $4 \%$ & $n=169$ & $3 \%$ \\
\hline COPD & $n=393$ & $3 \%$ & $n=155$ & $3 \%$ \\
\hline Malignant neoplasm of oesophagus & $n=272$ & $2 \%$ & $n=145$ & $3 \%$ \\
\hline Status epilepticus & $n=318$ & $2 \%$ & $n=80$ & $2 \%$ \\
\hline Bowel perforation & $n=305$ & $2 \%$ & $n=106$ & $2 \%$ \\
\hline Acute pancreatitis & $n=192$ & $1 \%$ & $n=83$ & $2 \%$ \\
\hline Diabetic ketoacidosis & $n=217$ & $2 \%$ & $n=57$ & $1 \%$ \\
\hline Asthma attack in new or known asthmatic patients & $n=201$ & $2 \%$ & $n=83$ & $2 \%$ \\
\hline Ventricular tachycardia or fibrillation & $n=169$ & $1 \%$ & $n=75$ & $2 \%$ \\
\hline Acute myocardial infarction & $n=175$ & $1 \%$ & $n=66$ & $1 \%$ \\
\hline
\end{tabular}

Change analysis was performed for each instrument, including only those patients who reached the caseness threshold at one but not both time points (Fig. 2). The magnitude of this change was significant in the majority of cases: $76 \%$ and $81 \%$ of patients changed their anxiety/depression score by more than 3 points ( $3 / 22$ patients ( $14 \%$ change)). A further $84 \%$ of patients experienced a change of 7 of more points $(7 / 69$ patients (10\% change) $)$ in their PCL-C score.

\section{Concurrent psychopathology}

Figure 3 shows the concurrent caseness of anxiety, depression and PTSD in individual patients. Of respondents, $55.2 \%(2731 / 4943)$ met caseness thresholds for at least one of the three conditions at either 3 or
12 months: $35.8 \%(1770 / 4943)$ met caseness thresholds for more than one psychopathological issue. Meeting caseness thresholds for PTSD alone was the least common (36 participants, $0.7 \%$ of those reporting psychopathological issues).

\section{Survival}

Figure 4 shows the 24-month survival of patients who responded at 3 or 12 months, dichotomised by those who had reported caseness for depression at either time point and those that had not. Survival data to 24 months were available for patients in phase 1 and 2 of the study, whilst phase 3 mortality data ended at 12 months (Additional file 8). Patients with a HADS-D $\geq 8$ experienced a 


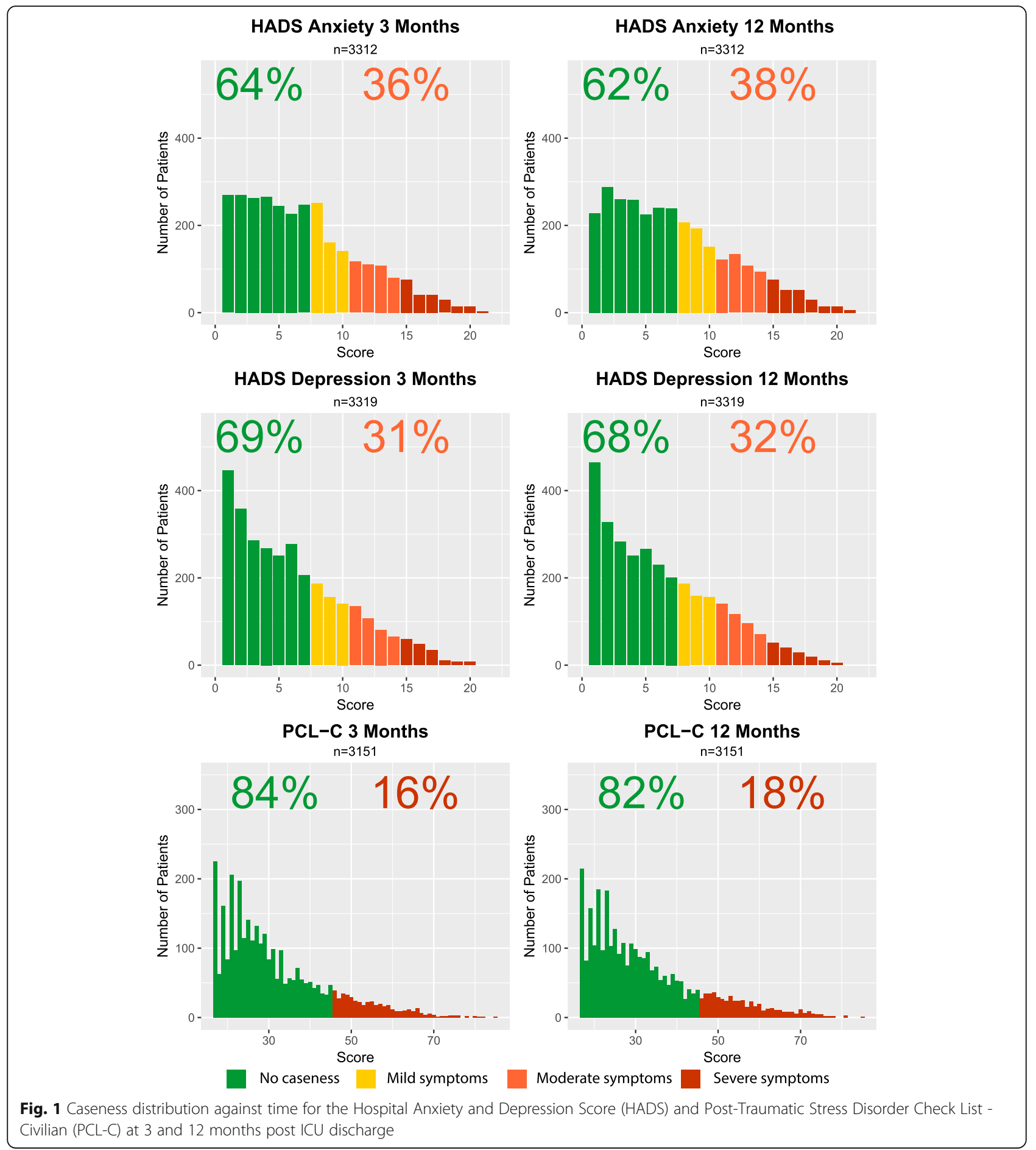

higher likelihood of death in the first 2 years after discharge from ICU $(p<0.001)$. Kaplan-Meier (KM) curves for anxiety and PTSD caseness are presented in Additional files 9 and 10 and do not demonstrate a significant difference between the respective groups.

Results from the univariable and multivariable Cox proportional hazards modelling are reported in
Additional file 11. Multivariable modelling was conducted for each type of caseness individually (models 1-3) and for all simultaneously (model 4). The hazard ratios in the multivariable models have been adjusted for all other variables in the model. Figure 5 is a graphical representation of the hazard ratios and confidence intervals from the final multivariable model 

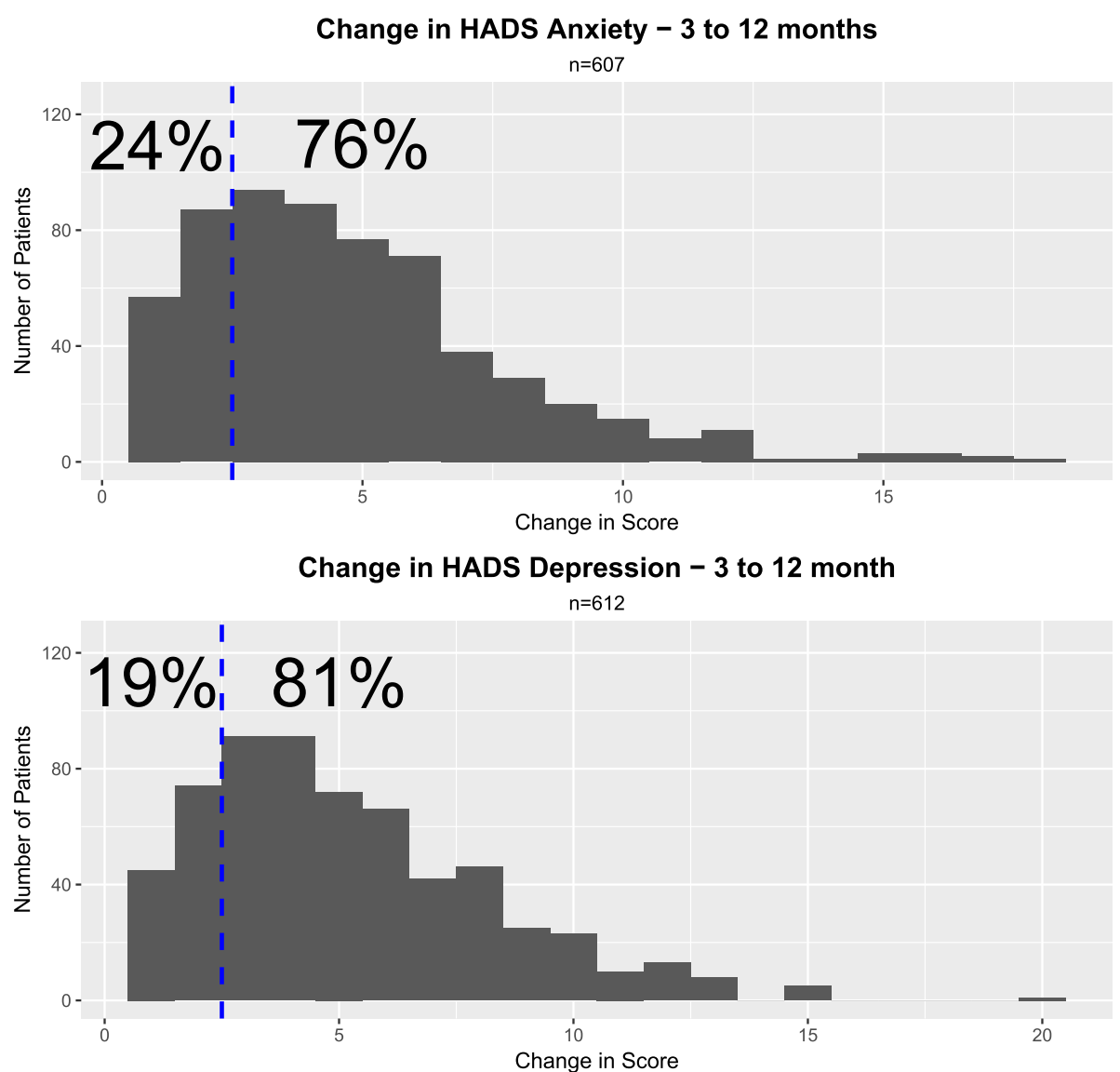

Change in PCL-C - 3 to 12 month

$n=380$

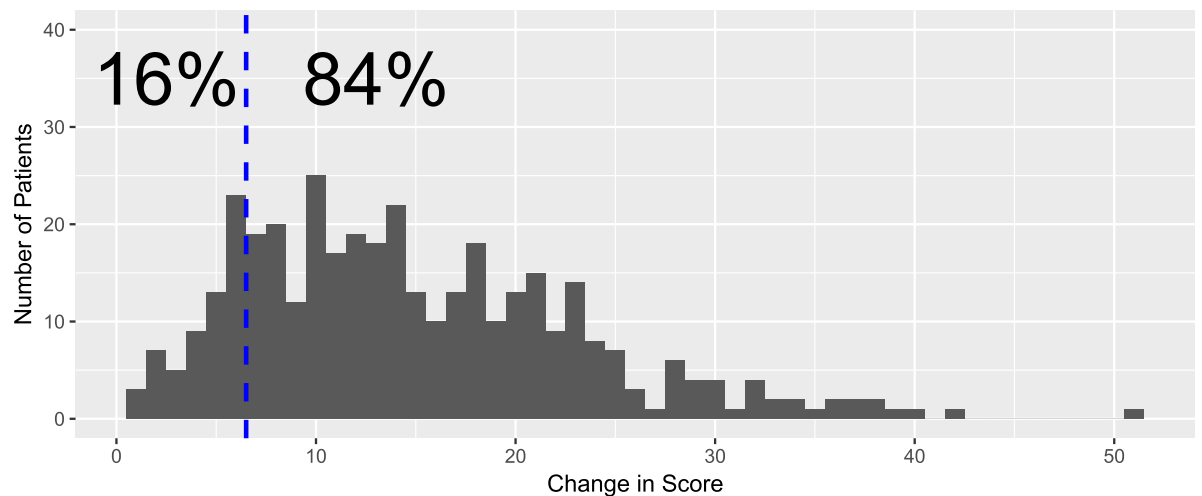

Fig. 2 Analysis of change in the Hospital Anxiety and Depression Score (HADS)/Post-Traumatic Stress Disorder Check List - Civilian (PCL-C) in responders between 3 and 12 months post ICU discharge

(Additional file 11, model 4). This model takes the fact that some patients may demonstrate symptoms for one or more of anxiety, depression or PTSD into account (as seen in Fig. 3). Adjusted for age, sex, illness severity and the presence of other psychopathological issues, those meeting caseness thresholds for depression were around $50 \%$ more likely to die in the 2 years following discharge than those not meeting caseness (hazard ratio 1.47 , 95\% CI 1.19-1.80).

\section{Discussion}

\section{Key results}

We present the largest multicentre postal survey of self-reported anxiety, depression and PTSD of ICU survivors to date. A high burden of post-ICU psychopathological issues was reported with over half of respondents meeting caseness thresholds for anxiety, depression or PTSD. A high degree of symptom concurrency between these three conditions was observed. 
Responders at 3 or 12 months $(n=4943)$

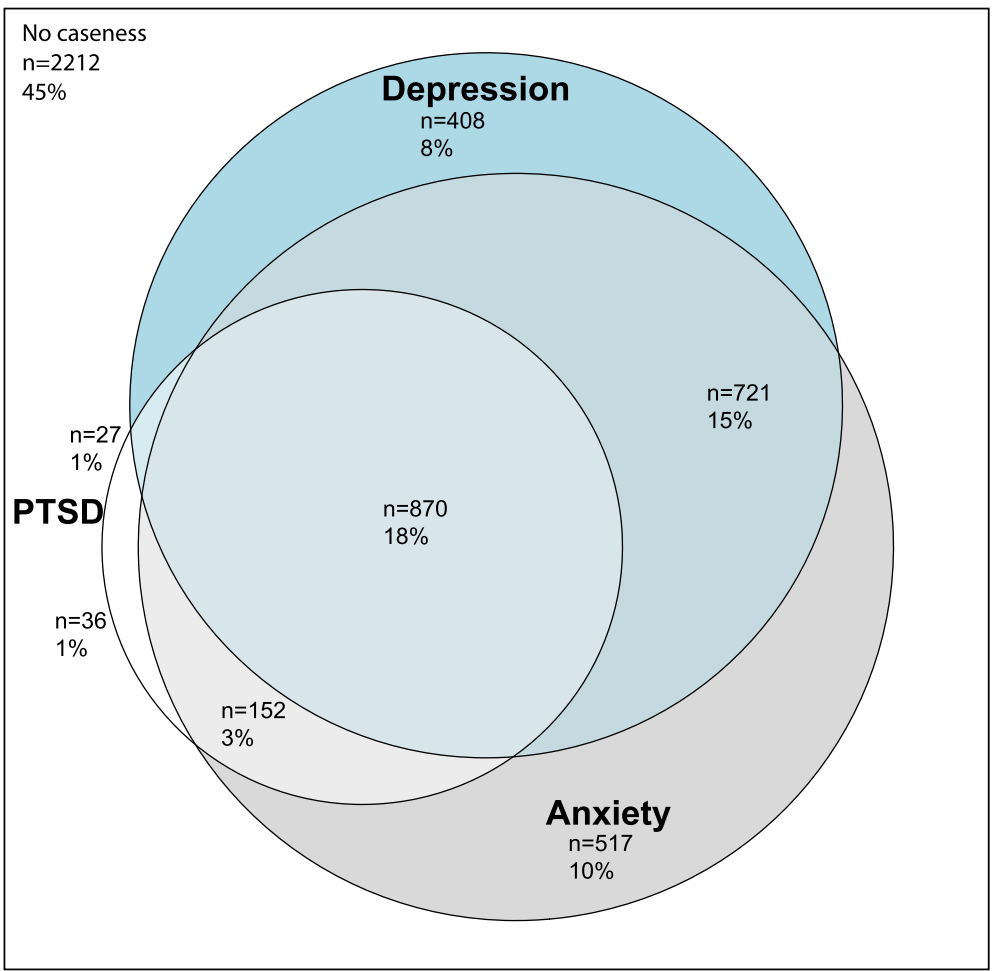

Fig. 3 Cross over in caseness - anxiety/depression and post-traumatic stress disorder (PTSD) caseness amongst responders
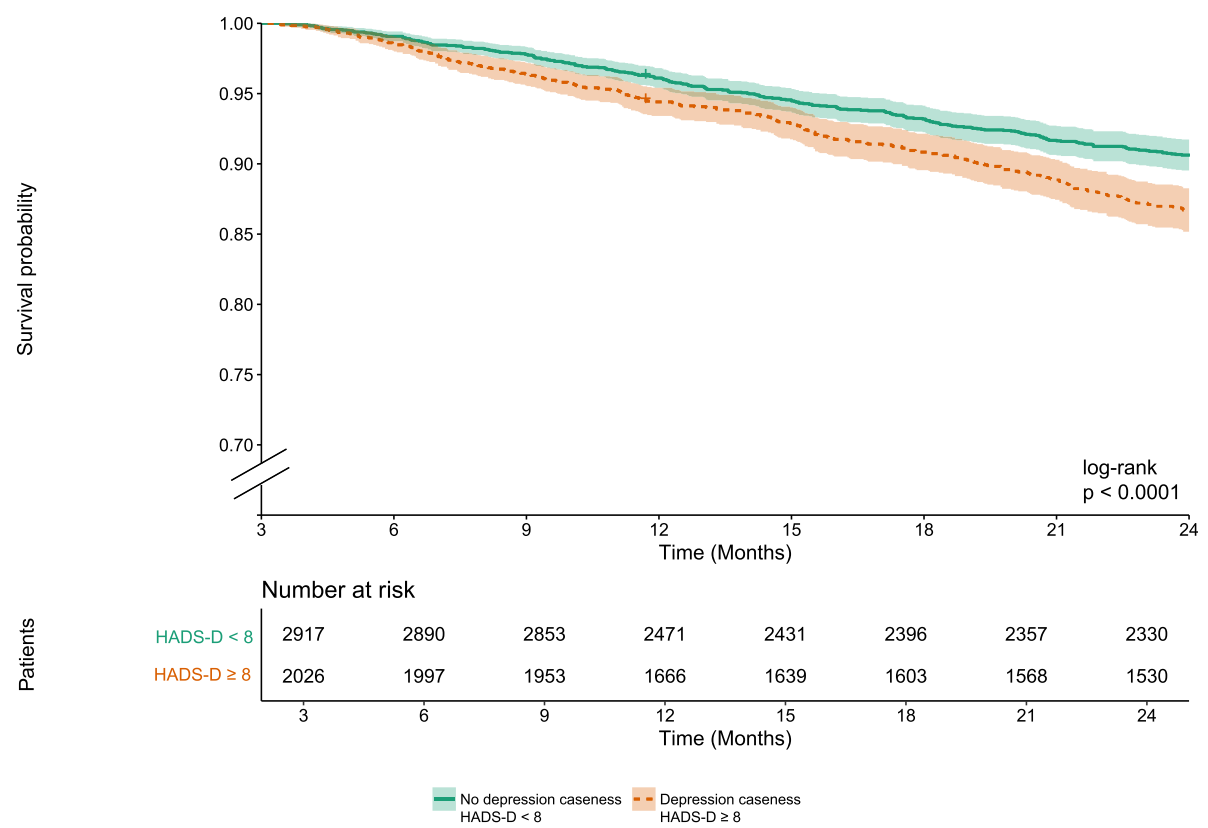

Fig. 4 Survival versus depression (2 years) - Kaplan-Meier curve of 2-year survival amongst responders grouped by depression caseness. HADS, Hospital Anxiety and Depression Score 


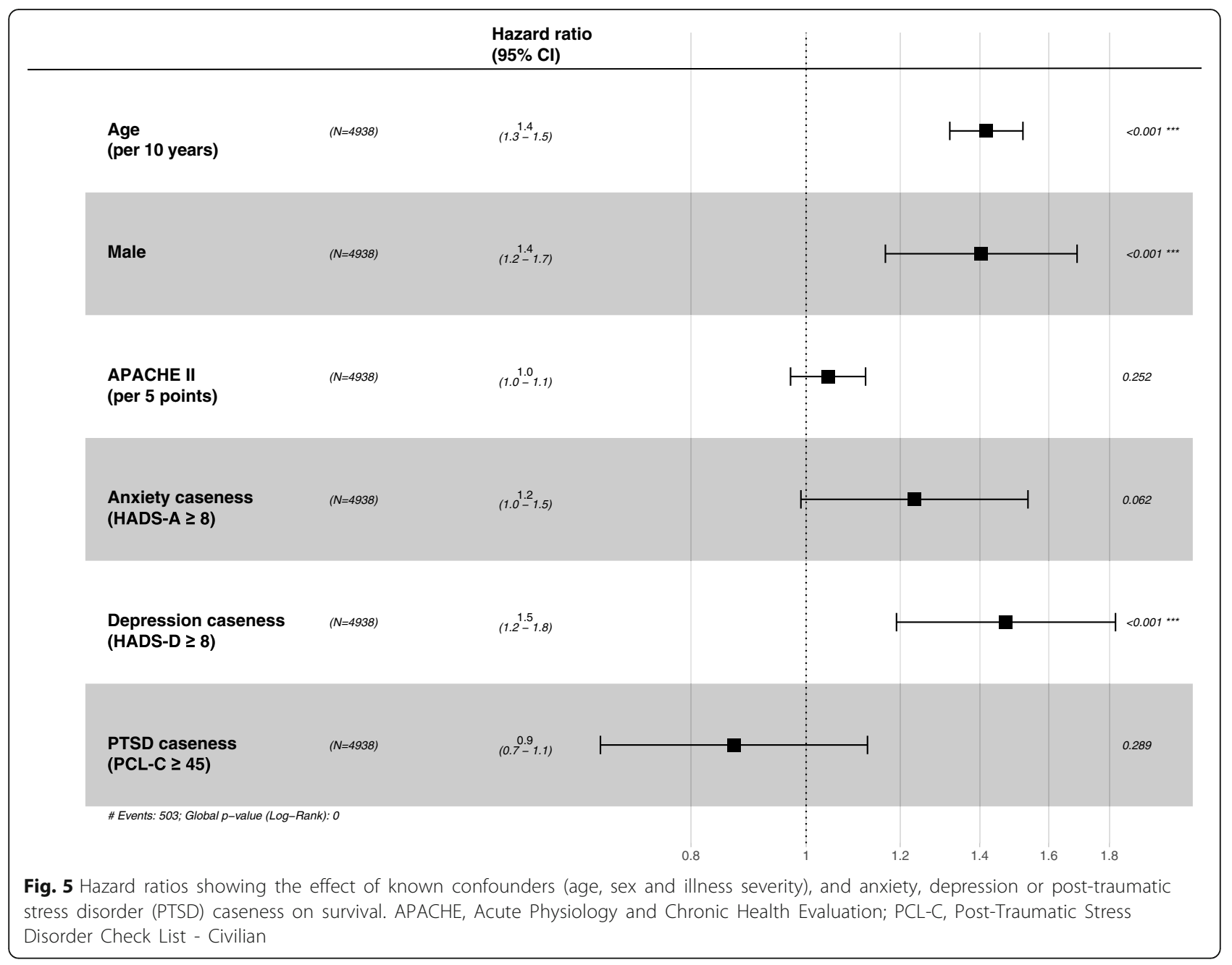

Especially interesting is the relatively low incidence of isolated PTSD, occurring in fewer than 1 in 20 individuals reporting any form of suspected psychopathological issue. Conversely, symptoms of anxiety occurred in $83 \%$ of individuals reporting any psychopathological issue. A strong association between self-reported symptoms of depression and mortality has been demonstrated in ICU survivors. When other known associations are adjusted for (age, male sex, illness severity), patients with depressive symptoms are nearly $50 \%$ more likely to die during the first 24 months after leaving ICU than those without depression caseness.

\section{Interpretation and generalisability}

Our study has shown that symptoms of anxiety, depression or PTSD occur in half of patients who respond to a postal questionnaire after treatment on a UK ICU, with nearly a third displaying symptoms of two or more conditions at 3 or 12 months following ICU discharge in the UK. This is comparable with incidence of persistent psychopathological issues in longer-term follow-up studies of survivors of ARDS where both the overall incidence of anxiety, depression and PTSD and co-occurrence of psychopathological issues are similar [26]. It is noteworthy that this pattern and prevalence of psychopathological issues extends across a UK general adult ICU population. Clinicians involved in the follow up and assessment of all survivors of ICU should be aware of the co-occurrence of psychopathological conditions as part of PICS. In this cohort, PTSD rarely occurs in isolation, instead strongly co-occurring with anxiety. Pre-existing anxiety has been demonstrated as a risk factor for developing PTSD in urban populations [27]; on meta-analysis a pre-existing psychopathological condition was the only pre-ICU factor that was consistently associated with PTSD symptoms. Conversely, PTSD symptomatology is strongly correlated with both post-ICU anxiety [3] and co-morbid depression post critical illness [28]. It seems logical that anxiety can lead to PTSD. However, these data lack any detail on pre-existing psychological status.

Our study discovered a previously unknown association between symptoms of depression and increased mortality 
for 2 years following ICU discharge. The association persists when adjusted for age, illness severity, sex and the presence of other psychopathological issues. Although depressive symptomatology is associated with increased mortality in other populations, it is with less magnitude than the $47 \%$ increased risk we have demonstrated following ICU discharge. For comparison, a large US study in a general veterans' population estimates that diagnosed depression is associated with $17 \%$ greater hazard of all-cause mortality at 3 years [11]. This study also demonstrates that depression is associated with higher mortality rates for common medical conditions such as heart disease, respiratory illness, cerebrovascular disease, accidents, diabetes mellitus and hypertension. It remains unclear whether clinicians are overlooking the screening and treatment of depression in favour of treating chronic medical conditions or whether there is a biological association between chronic illness and depression. In the post-ICU population, the observed association between depression and mortality could be in part explained by the severity of chronic illness both pre-discharge and post-discharge factors we did not adjust for in this study.

However, to the best of our knowledge, an association between depression and an increased rate of mortality after discharge from ICU has not been demonstrated previously. Depression could be a factor not previously considered in post-ICU survival. Given the incidence of depression amongst survivors, symptoms of this condition should be detected and managed during the time following ICU in primary care and in ICU follow-up clinics.

\section{Limitations}

The main limitation of our postal survey is the relatively low response rate that potentially limits the applicability of these results. It is not possible using these data to infer the reason for non-response and as a consequence there is a potential selection/information bias in the results This postal survey followed best practice guidance and an RCT performed as part of this study demonstrated that a reduced questionnaire burden did not improve the response rate [16]. Failure of the postal network to return questionnaires is highly unlikely, however we cannot exclude the possibility that patients discharged from hospital may not initially return to their permanent address, limiting the delivery of questionnaires to recipients [29]. However, it is unlikely to be the universal reason. A significant proportion (22\%) received a postal questionnaire and were able to contact the study in order to explicitly decline consent. There is clearly a significant group of people that are physically and mentally capable of returning a questionnaire but explicitly do not wish to take part in this form of research.
We suggest that the limited response rate is predominantly a methodological limitation of undertaking a postal survey in a post critical illness population in the UK. KM survival analysis of the 3-24-month period post ICU discharge included those meeting the caseness threshold at 3 or 12 months. There is the potential that including the 12-month respondents added bias. Reporting depression at 12 months would exclude those who had died in the preceding period and bias towards fewer deaths in those with depression.

A postal survey can only be used to calculate the prevalence of disease caseness rather than true clinical diagnostic rates. The validity of our findings is therefore reliant on the psychometric properties of the instruments used. The instruments employed here have been validated in similar cohorts. HADS [30] has been found to perform well in assessing the symptom severity and caseness of anxiety disorders and depression in patients with somatic symptoms and patients in psychiatric or primary care as well as in the general population [18, 31]. The psychometric properties of HADS have been evaluated in survivors of acute lung injury and its use has been suggested as part of a core-outcomes set for future clinical trials $[32,33]$. Interpretation is based primarily on the cut-off scores. The authors of HADS suggested a 4-tier system (normal $<=7$, mild 8-10, moderate 11-14 and severe 15-21) [19]. Subsequent studies have suggested a score of $\geq 8$ provides sensitivity and specificity of 0.80 for both HADS anxiety and depression [34] although, certain conditions are known to affect the sensitivity and specificity e.g. traumatic brain injury [35].

This study had limited access to pre-morbid conditions, specifically pre-existing psychological and psychiatric conditions. Patients with pre-existing psychopathological conditions are at higher risk of both developing new symptoms and worsening existing problems following treatment in the ICU. In addition, it is possible that a phenotype exists where pre-morbid sufferers of anxiety/ depression/PTSD are at a higher risk of developing critical illness. Future studies should collect pre-morbid psychological history in order to explore this hypothesis further.

The existing literature reports on the use of a variety of PTSD instruments, with the most popular being the Impact Event Scale (IES) [25]. Our study chose PCL-C above IES as the latter lacks sufficient sensitivity and specificity in the ICU population [36, 37]. Correlation of 0.93 between the total PCL-C score and structured interview with the Clinician-Administered PTSD Scale (CAPS) has been demonstrated (diagnostic efficiency 0.9 ) [38]. A score of 45 or greater on the PCL-C has been recommended as a cut-off for high PTSD symptom load, with sensitivity of 0.60 and specificity of 0.99 
for diagnosing PTSD when compared with a structured clinical interview for the Diagnostic and Statistical Manual of Mental Health Disorders (DSM-IV) in survivors of breast cancer [20].

This study reports results from 3 different phases of the ICON study. There were small differences in the methodologies between studies (for a summary see Additional file 1). However, recruitment criteria and the instruments used to assess the psychopathological issues were identical, minimising the risks of including all 3 phases in this analysis.

\section{Future considerations}

Following these results, future work should focus on describing the population that fails to respond to postal questionnaire. This should include determining whether study participants are in long-term care facilities, home care or otherwise incapacitated, as well as exploring some of the reasons why those who are physically and mentally able to respond choose not to. In addition, the utility of the postal questionnaire in detecting undiagnosed psychopathological issues should be evaluated and the treatments that result from detection described. This study strongly emphasises that future work should evaluate all forms of psychopathological conditions simultaneously, rather than focusing on a single specific condition. Collecting data on pre-morbid psychological and medical co-morbidities would also be essential in terms of understanding the risk factors for developing PICS, as current illness severity scoring and organ support information is clearly insufficient when it comes to understanding which individuals are at greatest risk.

\section{Conclusions}

Half of those who respond to postal questionnaire following treatment on ICU in the UK report significant symptoms of anxiety, depression or PTSD at 3 and 12 months after discharge. When symptoms of one psychological condition are present, there is a $64 \%$ chance they will co-occur with symptoms of another. Survivors of critical illness who report symptoms of depression have an increased risk of dying in the 2 years following discharge from ICU.

\section{Additional files}

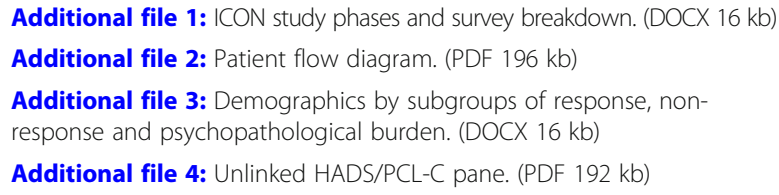

Additional file 5: HADS responses. (DOCX $18 \mathrm{~kb}$ )

Additional file 6: $\mathrm{PCL}-\mathrm{C}$ responses. (DOCX $17 \mathrm{~kb}$ )

Additional file 7: Changes in caseness against time - contingency tables showing the number and percentage of responders meeting the respective caseness thresholds at 3 and 12 months post ICU discharge. (DOCX $14 \mathrm{~kb}$ )

Additional file 8: $\mathrm{KM}$ - entire population. (PDF $152 \mathrm{~kb}$ )

Additional file 9: KM - anxiety. (PDF $159 \mathrm{~kb}$ )

Additional file 10: KM - PTSD. (PDF $156 \mathrm{~kb}$ )

Additional file 11: Cox proportional hazard regression analysis on factors affecting 2-year survival. (DOCX 14 kb)

\section{Abbreviations}

APACHE: Acute Physiology and Chronic Health Evaluation; ARDS: Acute respiratory distress syndrome; EQ-5D-3 L: EuroQol group 5 Dimensions 3 level; HADS: Hospital Anxiety and Depression Score; ICNARC: Intensive Care National Audit \& Research Centre; ICON: Intensive Care Outcome Network; ICU: Intensive care unit; MRIS: Medical Research Information Service; NHS: National Health Service; NIHR: National Institute for Health Research; ONS: Office of National Statistics; PCL-C: Post-Traumatic Stress Disorder Check List - Civilian; PTSD: Post-traumatic stress disorder; REC: Research Ethics Committee; SF36v2: Short Form 36 version 2; SWAT: Study within a trial; UK: United Kingdom

\section{Acknowledgements}

Peter Watkinson was supported by the National Institute for Health Research (NIHR) Biomedical Research Centre, Oxford.

Robert Hatch is funded by an NIHR National Academic Clinical Fellowship. This paper presents independent research funded by the NIHR. The views expressed are those of the author(s) and not necessarily those of the NHS, the NIHR or the Department of Health.

We thank Mr Barry Williams for more than fulfilling the role of patient representative on our trial steering committee. We are grateful to all those patients who kindly took part in our study whilst recovering from a significant period of ill health.

We thank Susan Dutton, Lead Medical Statistician for Oxford Clinical Trials Research Unit and Tatjana Petrinic, Outreach Librarian, University of Oxford Cairns Library, John Radcliffe Hospital Oxford.

We would like to thank the following Intensive Care Units for taking part in this study: Bedford Hospital NHS Trust; Diana Princess of Wales Hospital, Grimsby; George Eliot Hospital NHS Trust, Nuneaton; Gloucester Hospital; Great Western Hospitals NHS Trust, Swindon; Hartlepool Hospital; Hemel Hempstead General Hospital; Hillingdon Hospital; James Cook University Hospital, Middlesbrough; James Paget Hospital, Great Yarmouth; Luton and Dunstable University Hospital; Medway NHS Foundation Trust, Gillingham; Nevill Hall Hospital, Abergavenny; New Cross Hospital, Wolverhampton; North Tees Hospital, Stockton-on-Tees; Oxford University Hospitals NHS Foundation Trust; Poole Hospital; Queen Mary's Hospital, Sidcup; Royal Hampshire County Hospital, Winchester; Royal Lancaster NHS Trust; Russells Hall Hospital, Dudley; Southend NHS Trust, Westcliff-on-Sea; Stafford Hospital; The Royal Bournemouth and Christchurch NHS Trust; Trafford Hospital; Watford General Hospital. We would also like to thank the Intensive Care National Audit \& Research Centre (ICNARC) for providing data on patients included in the study, Dr Gillian Fortune for her much needed psychology input and Professor Nicholas Mays (all members of the ICON steering group). Finally, we would like to thank Verity Westgate for proof-reading and improving the wording of the manuscript.

\section{Funding}

The Intensive Care Outcomes Network study was funded by the Intensive Care Foundation and the BUPA Foundation. Peter Watkinson is supported by the Oxford Biomedical Research Centre.

\section{Availability of data and materials}

The anonymised dataset is held by the corresponding author and data may be made available in part for secondary analysis by third parties, access will 
be considered on a case by case basis. We did not obtain consent for data sharing, so we will only make anonymised data available.

\section{Authors' contributions}

$\mathrm{RH}$ helped design the study, developed and maintained the systems for data collection, prepared the data for analysis, undertook data analysis and wrote the paper. DY conceived the study, oversaw trial management and revised the paper. VB helped design the study, undertook trial management and revised the paper. DH took part in data analysis and study oversight, undertook the data linkage and revised the paper. JG helped design the study protocol, contributed to trial management and revised the paper. PW took part in managing the trial, oversaw and took part in data analysis, revised the paper, and is the guarantor. Peter Watkinson affirms that this manuscript is an honest, accurate, and transparent account of the study being reported; that no important aspects of the study have been omitted; and that any discrepancies from the study as planned (and, if relevant, registered) have been explained. The Oxford Radcliffe Hospitals NHS Trust (now the Oxford University Hospitals NHS Foundation Trust) sponsored the study but had no part in the conduct or analysis of the study. All authors read and approved the final manuscript.

\section{Ethics approval and consent to participate}

This study was granted national ethics approval. Phases 1 and 2 - Oxford Research Ethics Committee B (REC 06/Q1605/17), phase 3 - South Central Berkshire REC (11/SC/0172). Informed written consent to participate in the study was obtained from all patients who chose to respond to a questionnaire.

\section{Consent for publication}

Not applicable.

\section{Competing interests}

All authors have completed the ICMJE uniform disclosure form at www.icmje.org/coi_disclosure.pdf and declare no financial relationships with any organisations that might have an interest in the submitted work in the previous three years; no other relationships or activities that could appear to have influenced the submitted work.

\section{Publisher's Note}

Springer Nature remains neutral with regard to jurisdictional claims in published maps and institutional affiliations.

\section{Author details}

${ }^{1} \mathrm{NIHR}$ Academic Clinical Fellow in Intensive Care Medicine, Oxford Deanery, Oxford, UK. ${ }^{2}$ Professor of Intensive Care Medicine, University of Oxford, Oxford, UK. ${ }^{3}$ OCTRU Hub Manager, University of Oxford, Oxford, UK. ${ }^{4}$ Consultant in Intensive Care Medicine, Oxford University Hospitals NHS Foundation Trust, Oxford, UK. ${ }^{5}$ Head Statistician, Intensive Care National Audit \& Research Centre (ICNARC), London, UK. ${ }^{6}$ Associate Professor of Intensive Care Medicine, Oxford University Hospitals NHS Trust, Oxford, UK. ${ }^{7}$ Kadoorie Centre for Critical Care Research and Education, University of Oxford, Level 3, John Radcliffe Hospital, Headley Way, Oxford OX3 9DU, UK.

Received: 14 June 2018 Accepted: 9 October 2018 Published online: 23 November 2018

\section{References}

1. Desai SV, Law TJ, Needham DM. Long-term complications of critical care. Crit Care Med. 2011;39:371-9. https:/doi.org/10.1097/CCM.0b013e3181fd66e5.

2. Judy E. Davidson, RN, CNS, DNP, FCCM; Ramona O. Hopkins, PhD; Deborah Louis, RN, MSN; Theodore J. Iwashyna, MD P (2013) Post-intensive care syndrome. In: Soc Crit Care Med http://www.myicucare.org/Thrive/Pages/ Post-intensive-Care-Syndrome.aspx. Accessed 12 Feb 2018.

3. Nikayin S, Rabiee A, Hashem MD, et al. Anxiety symptoms in survivors of critical illness: a systematic review and meta-analysis. Gen Hosp Psychiatry. 2016:43:23-9.

4. Wade DM, Howell DC, Weinman JA, et al. Investigating risk factors for psychological morbidity three months after intensive care: a prospective cohort study. Crit Care. 2012;16. https://doi.org/10.1186/cc11677.

5. Samuelson KAM, Lundberg D, Fridlund B. Stressful memories and psychological distress in adult mechanically ventilated intensive care patients - a 2-month follow-up study. Acta Anaesthesiol Scand. 2007:51: 671-8. https://doi.org/10.1111/j.1399-6576.2007.01292.x.

6. Rattray JE, Johnston M, Wildsmith JAW. Predictors of emotional outcomes of intensive care. Anaesthesia. 2005;60:1085-92. https://doi.org/10.1111/j. 1365-2044.2005.04336.x

7. Cuthbertson BH, Rattray J, Campbell MK, et al. The PRaCTICaL study of nurse led, intensive care follow-up programmes for improving long term outcomes fromcritical illness: a pragmatic randomised controlled trial. BMJ. 2009;339:1016. https://doi.org/10.1136/bmj.b3723.

8. Myhren $\mathrm{H}$, Ekeberg $\varnothing$, Tøien $\mathrm{K}$, et al. Posttraumatic stress, anxiety and depression symptoms in patients during the first year post intensive care unit discharge. Crit Care. 2010;14:R14. https://doi.org/10.1186/cc8870.

9. Rabiee A, Nikayin S, Hashem MD, et al. Depressive symptoms after critical illness: a systematic review and meta-analysis. Crit Care Med. 2016;44:174453. https://doi.org/10.1097/CCM.0000000000001811.

10. Wright JC, Plenderleith $L, S$ a R. Long-term survival following intensive care: subgroup analysis and comparison with the general population. Anaesthesia. 2003;58:637-42. https://doi.org/10.1046/j.1365-2044.2003.03205.x.

11. Zivin $\mathrm{K}$, Yosef M, Miller EM, et al. Associations between depression and allcause and cause-specific risk of death: a retrospective cohort study in the Veterans Health Administration. J Psychosom Res. 2015;78:324-31. https:// doi.org/10.1016/j.jpsychores.2015.01.014

12. Cuijpers P, Smit F. Excess mortality in depression: a meta-analysis of community studies. J Affect Disord. 2002;72:227-36. https://doi.org/10.1016/ S0165-0327(01)00413-X.

13. Davydow DS, Gifford JM, Desai SV, et al. Posttraumatic stress disorder in general intensive care unit survivors: a systematic review. Gen Hosp Psychiatry. 2008;30:421-34. https://doi.org/10.1016/j.genhosppsych.2008.05.006.

14. von Elm E, Altman DG, Egger M, et al. The Strengthening the Reporting of Observational Studies in Epidemiology (STROBE) statement: guidelines for reporting observational studies. PLoS Med. 2007;4:e296. https://doi.org/10. 1371/journal.pmed.0040296.

15. Griffiths JA, Morgan K, Barber VS, Young JD. Study protocol: The Intensive Care Outcome Network ('ICON') study. BMC Health Serv Res. 2008;8. https:// doi.org/10.1186/1472-6963-8-132.

16. Hatch $R$, Young D, Barber $V$, et al. The effect of postal questionnaire burden on response rate and answer patterns following admission to intensive care: a randomised controlled trial. BMC Med Res Methodol. 2017;17. https://doi.org/10.1186/s12874-017-0319-3.

17. Intensive Care Society. A Guide for critical care settings standards for critical incident reporting in critical care; 2005. p. 37.

18. Bjelland I, Dahl AA, Haug T, Neckelmann D. The validity of the Hospital Anxiety and Depression Scale. J Psychosom Res. 2002;52:69-77. https://doi. org/10.1016/S0022-3999(01)00296-3.

19. Snaith RP, Zigmond AS (1994) The Hospital Anxiey and Depression Scale with the Irritability-depression-anxiety Scale and the Leeds Situational Anxiety Scale: manual.

20. Andrykowski MA, Cordova MJ, Studts JL, Miller TW. Posttraumatic stress disorder after treatment for breast cancer: prevalence of diagnosis and use of the PTSD Checklist - Civilian Version (PCL-C) as a screening instrument. J Consult Clin Psychol. 1998;66:586-90. https://doi.org/10.1037/0022-006X.66.3.586.

21. Harrison DA, Brady AR, Rowan K. Case mix, outcome and length of stay for admissions to adult, general critical care units in England, Wales and Northern Ireland: the Intensive Care National Audit \& Research Centre Case Mix Programme Database. Crit Care. 2004;9(S1). https://doi. org/10.1186/cc3745.

22. NHS Digital (2016) NHS digital - summary care records. https://www.digital. nhs.uk/summary-care-records. Accessed 4 Oct 2017.

23. R core team. R: A language and environment for statistical computing. Vienna: R Found. Stat. Comput; 2017.

24. Knaus WA, Draper EA, Wagner DP, Zimmerman JE. APACHE I: a severity of disease classification system. Crit Care Med. 1985;13:818-29. https:// doi.org/10.1097/00003246-198510000-00009.

25. Parker AM, Sricharoenchai T, Raparla S, et al. Posttraumatic stress disorder in critical illness survivors: a metaanalysis. Crit Care Med. 2015;43:1121-9. https://doi.org/10.1097/CCM.0000000000000882.

26. Bienvenu OJ, Friedman LA, Colantuoni E, et al. Psychiatric symptoms after acute respiratory distress syndrome: a 5-year longitudinal study. Intensive Care Med. 2018;44:38-47. https://doi.org/10.1007/s00134-017-5009-4.

27. Breslau N, Davis GC, Andreski P, Peterson E. Traumatic events and posttraumatic stress disorder in an urban population of young adults 
lifetime prevalence of exposure to traumatic events. Arch Gen Psychiatry. 1991;48:216-22.

28. Paparrigopoulos T, Melissaki A, Tzavellas E, et al. Increased co-morbidity of depression and post-traumatic stress disorder symptoms and common risk factors in intensive care unit survivors: a two-year follow-up study. Int J Psychiatry Clin Pract. 2014;18:25-31. https:/doi.org/10.3109/13651501.2013.855793.

29. Gehlbach BK, Salamanca VR, Levitt JE, et al. Patient-related factors associated with hospital discharge to a care facility after critical illness. Am J Crit Care. 2011;20:378-86. https://doi.org/10.4037/ajcc2011827.

30. Zigmond AS, Snaith RP. The Hospital Anxiety and Depression Scale. Acta Psychiatr Scand. 1983;67:361-70. https://doi.org/10.1111/j.1600-0447.1983. tb09716.x

31. Herrmann C. International experiences with the Hospital Anxiety and Depression Scale-a review of validation data and clinical results. J Psychosom Res. 1997:42:17-41. https://doi.org/10.1016/S00223999(96)00216-4.

32. Jutte JE, Needham DM, Pfoh ER, Bienvenu OJ. Psychometric evaluation of the Hospital Anxiety and Depression Scale 3 months after acute lung injury. J Crit Care. 2015;30:793-8. https://doi.org/10.1016/j.jcrc.2015.04.006.

33. Williamson PR, Altman DG, Blazeby JM, et al. Developing core outcome sets for clinical trials: issues to consider. Trials. 2012;13:132. https://doi.org/10. 1186/1745-6215-13-132.

34. Bjelland I, Dahl AA, Haug TT, Neckelmann D. The validity of the Hospital Anxiety and Depression Scale. An updated literature review. J Psychosom Res. 2002:52:69-77.

35. Dahm J, Wong D, Ponsford J. Validity of the depression anxiety stress scales in assessing depression and anxiety following traumatic brain injury. J Affect Disord. 2013;151:392-6. https://doi.org/10.1016/j.jad.2013.06.011.

36. Griffiths J, Fortune G, Barber V, Young JD. The prevalence of post traumatic stress disorder in survivors of ICU treatment: a systematic review. Intensive Care Med. 2007;33:1506-18. https://doi.org/10.1007/s00134-007-0730-z.

37. Joseph S. Psychometric evaluation of Horowitz's impact of event scale: a review. J Trauma Stress. 2000;13:101-13. https://doi.org/10.1023/A:1007777032063.

38. Blanchard EB, Jones-Alexander J, Buckley TC, Forneris CA. Psychometric properties of the PTSD checklist (PCL). Behav Res Ther. 1996;34:669-73. https://doi.org/10.1016/0005-7967(96)00033-2.

Ready to submit your research? Choose BMC and benefit from:

- fast, convenient online submission

- thorough peer review by experienced researchers in your field

- rapid publication on acceptance

- support for research data, including large and complex data types

- gold Open Access which fosters wider collaboration and increased citations

- maximum visibility for your research: over $100 \mathrm{M}$ website views per year

At $\mathrm{BMC}$, research is always in progress.

Learn more biomedcentral.com/submissions 